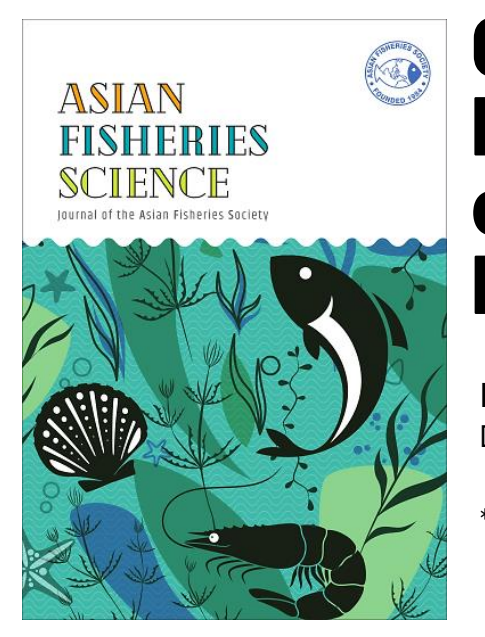

\title{
Critical Review of Methods Used in Published Studies of Susceptibility of Vibrio spp.; Lessons to Be Learnt
}

\author{
PETER SMITH*, SARAH EGAN \\ Department of Microbiology, School of Natural Science, National University of Ireland, Galway, Ireland \\ *E-mail: peter.smith@nuigalway.ie
}

(C)Asian Fisheries Society

ISSN: 0116-6514

E-ISSN: 2073-3720

https://doi.org/10.33997/j.afs.2020.33.S1.004

\section{Abstract}

The World Health Organization, the World Organisation for Animal Health, and the Food and Agriculture Organization of the United Nations recognises that the threat posed by the emergence of resistance to antimicrobials must be addressed using a One Health approach. To quantify the contribution of aquaculture to this global problem, it is essential that we generate data on the antimicrobial susceptibility of bacteria isolated from aquatic animals that is of sufficient quality. This paper presents a review of the quality of the data presented in 182 published papers on the antimicrobial susceptibility of various non-cholera Vibrio species isolated from aquatic environments. This review revealed that serious shortcomings either in the performance of the susceptibility tests or in the reporting of those tests occurred with an alarmingly high frequency. The majority of studies failed to provide sufficient details of the testing protocols they used and only a small percentage of the studies provided explicit evidence that they had used standardised susceptibility protocols. Although 203 studies reported frequencies of resistance in the isolates they studied, 185 of them either did not provided the criteria they used to determine resistance, used criteria that had not been validated or used criteria that were inappropriate. As a result of these shortcomings, it is difficult or impossible to compare the data that these papers have presented. It is argued that adopting a few simple rules in the design and reporting of susceptibility studies would, at little cost or effort, result in the production of papers that could make a significant contribution to our understanding of the issues involved in the use of antimicrobial agents in aquaculture.

Keywords: aquaculture, AMR, detection, analysis, CLSI

\section{Introduction}

In-vitro antimicrobial susceptibility tests are not robust and the quantitative data they produce is critically dependent on the details of the experimental protocols adopted in the performance of those tests (Smith, 2019). It is, therefore, essential that any report of susceptibility data is accompanied with a complete description of the testing protocol used to generate that data. When a report also includes an interpretation of the meaning of susceptibility data, it is also essential that the criteria and the sources of those criteria that are used to generate the meaning of the data are provided. However, reviews of published studies of antimicrobial susceptibility of isolates from humans (Turner and Ashley, 2019) and terrestrial animals (Schwarz et al., 2010) have demonstrated that shortcomings in the descriptions of the testing protocol and/or the interpretive criteria applied occur in these studies with a disturbing frequency.

In addition to these requirements the World Organisation for Animal Health (OIE) Aquatic Animal Health Code (OIE, 2018) has argued that, in order to ensure maximum international comparability, any programme for monitoring or surveillance of antimicrobial susceptibility of isolates from aquatic animals should be designed so that the data generated can be compared with data produced in other laboratories. Smith et al. (2013) have argued that to ensure maximum international comparability required by the Code, it is essential that standardised and internationally harmonised susceptibility test protocols be used to the greatest extent possible. 
They further recommend that the meaning of the quantitative data should be determined using internationally harmonised, consensus-based interpretive criteria when these are available.

This paper presents an analysis of the published literature on the antimicrobial susceptibility of noncholera Vibrio isolates. The analysis was performed to investigate how consistent the methods used in these studies were with current best-practice guidelines.

\section{Materials and Methods}

\section{Collection of the relevant published literature}

The first step in collecting papers that had been published on the susceptibility of non-cholera Vibrios was to use relevant keywords in Google Scholar. This identified an initial list of relevant papers. The second step was to examine those in the initial list for the papers they cited and the papers that cited them. Iteration of this process generated 190 papers. Copies of the full text were accessed for 182 of these. Some of these papers reported more than one study and in total the 182 papers provided details of 207 studies.

The papers originated from 39 different countries with the majority (55\%) originating from Asia, $20 \%$ from Europe, $15 \%$ from America, and $8 \%$ from Africa. The majority of the papers $(91 \%)$ had been published in the last 20 years and $62 \%$ published in the period 2008 to 2017 . The fact that the median citations that the papers received were 16 indicated that, generally, they had been widely consulted.

We do not believe that this process identified all relevant papers, but we do suggest that those accessed provide a reasonable overview of current practices in this field.

\section{Results and Discussion}

\section{General observations}

An observation that remains after reading 207 studies is that, for many of them, the methods used are inadequately or incompletely described and, on some occasions, incorrectly or inappropriately referenced. This may suggest that the authors have not given sufficient weight to the fact that the quantitative values generated from such tests are protocol dependent. Without full details of the methods used to generate them, it is impossible to establish the meaning that can be given to the quantitative data generated. The poor quality of the description of the methods used had a consequence for the analysis presented here. For many papers, it was not possible to determine how the quantitative data presented was obtained.

A second general observation is that the term 'resistant' was more or less universally used in the papers examined but its meaning was rarely defined. Silley (2012) has argued that much confusion has been generated by the variation in the meanings given to this word by different authors. It is strongly argued that, in order to improve communication, all workers in this field should adopt the use of the terminology suggested by Silley (2012).

\section{Which species were studied?}

In the 207 studies, five species were investigated most frequently. There were 88 studies that reported on the susceptibility of Vibrio parahaemolyticus, 72 on Vibrio alginolyticus, 50 on Vibrio harveyi, 27 on Vibrio vulnificus, and 19 on Vibrio anguillarum. In addition, 66 studies either reported the susceptibility of various other species of Vibrio or did not provide a specieslevel classification of the Vibrio isolates they studied.

\section{What media were used?}

Over $90 \%$ of the studies examined reported using either unmodified Mueller-Hinton agar (MHA), cationadjusted Mueller-Hinton broth (CAMHB), or these media supplemented with various concentrations of $\mathrm{NaCl}$. Analysis of the media used in these studies (Table1) showed that more than half had used media without additional $\mathrm{NaCl}$. This would suggest that the supplementation of MHA or CAMHB is not necessary for susceptibility testing of the majority of the Vibrionaceae.

\section{What incubation temperatures were used?}

Of the studies that reported the incubation temperature, the majority reported using temperatures at $\geq 28{ }^{\circ} \mathrm{C}$ for susceptibility testing of their Vibrios (Table 1). This would suggest that protocols that specify incubation at $28^{\circ} \mathrm{C}$ provided in VETO3-A (CLSI, 2006) and VET04-A2 (CLSI, 2014) would be suitable for these bacteria. Four of the most commonly studied Vibrio species (V. alginolyticus, V. harveyi, V. parahaemolyticus, and V. vulnificus) were reported as being capable of infecting humans. For these species, not surprisingly, a significant number of studies reported testing at $\geq 35{ }^{\circ} \mathrm{C}$. Thus, the protocols specifying incubation at $35{ }^{\circ} \mathrm{C}$ provided in M02-A12 (CLSI, 2015) and M07-A10 (CLSI, 2014) could be used for testing their susceptibility. It is, however, unlikely that $V$. anguillarum could be tested at this temperature.

\section{What methods were used?}

Most papers reported using either disc diffusion methods or minimum inhibitory concentration (MIC) methods, although a minority reported using both. In all of the papers collected, 150 studies that used disc diffusion methods, 50 that used MIC methods, and seven that used the hybrid E-test were reported. 


\begin{tabular}{llll}
\hline \multirow{2}{*}{ Species } & \multicolumn{2}{l}{ Media $^{\text {Temperatureb }}$} & \\
\cline { 2 - 4 } & No added NaCl & $\geq 35{ }^{\circ} \mathrm{C}$ & $\geq 28{ }^{\circ} \mathrm{C}$ \\
\hline V. alginolyticus & $56 \%$ & $44 \%$ & $80 \%$ \\
V. anguillarum & $40 \%$ & $0 \%$ & $76 \%$ \\
V. harveyi & $48 \%$ & $22 \%$ & $84 \%$ \\
V. parahaemolyticus & $68 \%$ & $65 \%$ & $95 \%$ \\
V. vulnificus & $76 \%$ & $35 \%$ & $85 \%$ \\
V. spp & $60 \%$ & $25 \%$ & $78 \%$ \\
\hline
\end{tabular}

apercentage of those studies that used Mueller-Hinton media.

${ }^{b}$ percentage of those that reported their incubation temperature.

${ }^{\circ}$. spp. includes unclassified isolates and miscellaneous species.

\section{Were international standard testing protocols used?}

In 103 of the 207 studies examined, insufficient details were provided to allow any identification of the source of the testing protocols used. Furthermore, 29 cited various books or papers as their source. However, 69 studies cited a Clinical and Laboratory Standards Institute (CLSI) document as the source of the protocol they used and an additional six papers stated that they used a CLSI protocol but provided no reference to a specific document.

Therefore, only $33 \%$ of the studies reported using an internationally standardised testing protocol and that approximately $50 \%$ failed to provide sufficient details of the source of the protocol they used, which is somewhat alarming. It is clear, however, that in the studies that did use a standard protocol, there was a clear preference for those published by CLSI.

\section{How many strictly adhered to the CLSI protocol they claimed to use?}

Of the studies that employed a disc diffusion method, 51 claimed to have used a standardised CLSI protocol. A detailed reading of the methods used in these studies revealed that 14 used a temperature other than that specified in the CLSI protocol and 16 used media with additional $\mathrm{NaCl}$. Consequently, only 31 (20 $\%$ ) of the 150 disc diffusion studies explicitly presented evidence that they used and adhered to a standard test protocol.

A similar situation was observed in the MIC studies. Of the 18 studies that claimed to have used a CLSI protocol, nine have modified the temperature or time. Therefore, only nine $(18 \%)$ of the 50 MIC studies explicitly presented evidence that they used a standard test protocol.

\section{How many studies reported compliance with the quality control criteria?}

Of the 31 disc studies that presented evidence of having used a standard CLSI protocol, 16 reported the use of a recommended reference strain as a quality control measure. Of these 16, only three reports cited an appropriate CLSI document as a source of the acceptable ranges for the reference strain they used. Additionally, there was one study that specified their results with the reference strain were within the acceptable range.

Of the nine MIC studies that presented evidence of having used a standard CLSI protocol, six reported the use of a recommended reference strain as a quality control measure. Of these six, only there was one report that cited an appropriate CLSI document as a source of the acceptable ranges for the reference strain they used. None reported the results they obtained with the reference strain they used.

Thus, very few of the studies provided evidence of their compliance with the quality control requirements of the standard protocol they used. It has to be borne in mind, however, that some authors may have considered that the statement where a standard protocol was used was sufficient to imply that the quality control measures specified in that protocol were also performed. Thus, full compliance with quality control may be more common than the examination of the relevant texts suggests.

\section{How many studies reported their raw quantitative data?}

Of the 207 studies examined, only four presented their quantitative data and five studies with histograms of these data. For $198(96 \%)$ studies, no quantitative data was made available. All the studies present estimates of the frequency of resistance, but only a minority reported the interpretive criteria used 
to categorise the isolates as resistant and hardly any provide the quantitative data to which these criteria were applied. The consequence of this is that it is not possible to recalculate the frequencies of resistance provided in these studies using the current international, consensus-based criteria.

\section{How many studies applied international consensus-based interpretive criteria?}

Interpretation of the meaning of raw quantitative data can be made by applying either epidemiological cutoff values (ECVs) or clinical breakpoints (CBs) to them. Both ECVs and CBs are species-specific and protocol-specific but differ in the data needed to set them and the meanings that can be given to the categories they delineate.

Setting ECVs is relatively easy. It requires only in-vitro susceptibility data of adequate quantity and quality. The application of an ECV allows the categorisation of an isolate as a fully susceptible member of their species (wild type or WT) or as manifesting a reduced susceptibility when compared to other members of its species (non-wild type or NWT). In contrast, setting CBs is difficult. As they aim to categorise isolates based on the most probable clinical outcome of specific therapy of a specified infected host their setting requires very extensive microbiological, clinical, and pharmacodynamics data. Very little of these data are available for aquatic infections. It has been argued that the time and effort needed to generate these data would mean that CBs, relevant to any aquatic animals, will not be available for some time (Smith, 2008).

At present, no ECVs have been set by CLSI for susceptibility data generated for any Vibrio species by any standard protocol. Concerning CBs, CLSI has published some breakpoints applicable to data for Vibrio species tested at $35^{\circ} \mathrm{C}$ on unmodified MuellerHinton media (CLSI, 2016). There are, however, two reasons why these breakpoints should be treated with caution.

The first is that there is little empirical evidence for them. The forward of the guideline that presents them states that "Users of the guideline should be aware that the very extensive microbiological, clinical, and pharmacodynamics databases normally used for setting breakpoints by CLSI do not exist for the collection of organisms described in this document". The CBs for Vibrio species presented in this document are, in fact, simply copied from the CBs presented for the Enterobacteriaceae in M100-A27 (CLSI, 2017), and very few cited papers provided evidence of their validity when applied to the Vibrio species isolated from aquatic animals.

The second follows the fact that of their nature, CBs are host specific. Those presented in the guideline
M45-A3 (CLSI, 2016) relate only to the prediction of the clinical outcomes of therapies of humans. They cannot, with any legitimacy, be applied to predicting the clinical outcome of infections of aquatic animals.

Of the 31 disc studies that are assumed to have used standardised CLSI test protocols, 17 cited an appropriate CLSI document as a source of the CBs they used to interpret the meaning of their data. One of these 17 was concerned with mortalities of abalone and, therefore, these CBs were not relevant to that study. Of the nine MIC studies that are assumed to have used appropriate CLSI test protocols, only two reports cited an appropriate CLSI document as a source of the CBs they used to interpret the meaning of their data.

Overall, only 18 ( $9 \%$ ) of the 207 studies examined explicitly provided evidence that standard test protocols had been used and relevant internationally harmonised interpretive criteria had been applied to the in-vitro data generated.

\section{The Way Forward}

It is possible to offer a few, relatively simple, recommendations that, if implemented, would go a long way to improving the cost-benefit ratio of studies of the susceptibility of bacteria isolated from aquatic animals.

1. Standard testing protocols are available for a large percentage of the bacterial species isolated from aquatic animals. When they are available, they should be used, and their procedures should be strictly adhered to. Compliance with the quality control procedures of these standard test protocols is an absolute requirement.

2. Data generated by susceptibility testing should be interpreted using internationally harmonised and consensus-based criteria when these are available. When they are unavailable, the meaning of the data should be established using ECVs calculated by an objective and statistically based method. For MIC data sets, two automatic validated statistical methods are available (ECOFFinder @ clsi.org/meetings/microbiology/ecoffinder/ and NRI@ http://www.bioscand.se/nri/).For disc data, there is only one automatic statistical method (NRI @ http://www.bioscand.se/nri//).

3. Reports of any susceptibility study should provide an accurate citation for the source of the test protocols used and present evidence of compliance with the quality control requirements of that protocol. They must also include or provide access to the unprocessed quantitative data generated in the study. 
A more comprehensive treatment of these recommendations has been provided by Smith (2020).

\section{Conclusion}

Improving our understanding of antimicrobial resistance (AMR) in aquaculture will require the consideration and comparison of a large number of susceptibility data sets collected from diverse environments. If these comparisons are to be made, the data sets must be commensurate (Smith et al., 2013). The 207 studies of the antimicrobial susceptibility of non-cholera Vibrio species analysed here have generated a truly vast amount of data from a geographically diverse set of environments. Due to either the lack of information provided about how they were obtained or variations in the protocols and interpretive criteria used to generate them, however very little of these data could be considered as commensurate. Therefore, it would seem to be an inescapable conclusion of the analysis presented here that very considerable time and effort have been expended but the gain in our understanding of AMR is disappointingly small.

\section{References}

CLSI. 2006. Method for antimicrobial disk susceptibility testing of bacteria isolated from aquatic animals; approved guideline VETO3-A. Clinical and Laboratory Standards Institute, Wayne, Pennsylvania.

CLSI. 2014. Methods for broth dilution susceptibility testing of bacteria isolated from aquatic animals. Approved guideline VETO4-A2, Clinical and Laboratory Standards Institute, Wayne, Pennsylvania.

CLSI. 2015. Performance standards for antimicrobial disc susceptibility tests. Approved standard $12^{\text {th }}$ Edition. CLSI document M02-A12. Clinical and Laboratory Standards Institute. Wayne, Pennsylvania.

CLSI. 2016. Methods for antimicrobial dilution and disc susceptibility testing of infrequently isolated or fastidious bacteria. CLSI guideline M45-A3. Clinical and Laboratory Standards Institute, Wayne, Pennsylvania.

CLSI. 2017. Performance standards for antimicrobial susceptibility testing. $27^{\text {th }}$ Edition. CLSI supplement M100-S27. Clinical and Laboratory Standards Institute; Wayne, Pennsylvania.

OIE. 2018. Aquatic animal health code (2018). Paris, World Organisation for Animal Health. www.oie.int/international-standardsetting/aquatic-code/access-online/(Accessed 16 September 2019).

Schwarz, S., Silley, P., Simjee, S., Woodford, N., van Duijkeren, E., Johnson, A.P., Gaastra, W., 2010. Assessing the antimicrobial susceptibility of bacteria obtained from animals. Journal of Antimicrobial Chemotherapy 65:601-604. https://doi.org/10.1093 ljac/dkq037

Silley, P. 2012. Susceptibility testing methods, resistance and breakpoints: what do these terms really mean? Revue Scientifique et Technique 31:33-41. https://doi.org/10.20506/rst.31.1.2097

Smith, P. 2008. A cost-benefit analysis of the application of pharmacokinetic/pharmacodynamic-based approaches to setting disc diffusion breakpoints in aquaculture: a case study of oxolinic acid and Aeromonas salmonicida. Aquaculture 284:2-18. https://doi.org/10.1016/j.aquaculture.2008.07.029

Smith, P. 2019. The performance of antimicrobial susceptibility testing programmes relevant to aquaculture and aquaculture products. FAO Fisheries and Aquaculture Circular No. 1191. FAO, Rome. 35 pp.
Smith, P. 2020. Eight rules for improving the quality of papers on the antimicrobial susceptibility of bacteria isolated from aquatic animals. Diseases of Aquatic Organisms 139:87-89. https://doi.org /10.3354/dao03476

Smith, P., Alday-Sanz V., Matysczak, J., Moulin, G., Lavilla-Pitogo, C.R. Prater, D. 2013. Monitoring and surveillance of antimicrobial resistance in microorganisms associated with aquatic animals. Revue Scientifique et Technique 32:583-593. https://doi.org $\underline{\text { /10.20506/rst.32.2.2237 }}$

Turner, P., Ashley, E.A. 2019. Standardising the reporting of microbiology and antimicrobial susceptibility data. The Lancet Infectious Diseases 19:1163-1164. https://doi.org/10.1016/S1473$\underline{3099(19) 30561-4}$ 